\title{
SPECTRA OF NILFLOWS
}

\author{
BY L. W. GREEN ${ }^{1}$ \\ Communicated by W. S. Massey, April 8, 1961
}

Recently L. Auslander, F. Hahn, and L. Markus [1] have examined an important class of dynamical systems, proving the existence of minimal, distal, nonequicontinuous flows on compact nilmanifolds. Their proof is nonconstructive, and gives no information on the measure-theoretic behavior of these flows. Since these flows are defined by the action of one parameter subgroups of the universal covering group of the nilmanifold, it is natural to ask whether the group representation approach of Gelfand-Fomin [3] and Mautner [4] can give more detailed information. The author has shown that this is the case. In fact, a complete qualitative description of the spectra of these flows can now be given, and one can tell precisely which one parameter subgroups give rise to minimal, ergodic flows.

Let $N$ be a connected, simply connected, nilpotent Lie group with discrete uniform subgroup $D$ and one parameter subgroup $\left\{\gamma_{t}\right\},-\infty$ $<t<\infty$. The flow on the nilmanifold $M=N / D$ is defined by $\phi_{t}(D n)$ $=D n \gamma_{t} . N$ itself acts transitively on $M$ in the same way, inducing a unitary representation $\gamma \rightarrow V(\gamma)$ of $N$ in the Hilbert space of functions on $M$, square summable with respect to the natural invariant measure. This representation must be expressible as a direct integral of irreducible unitary representations of $N$. Not all of these have been computed explicitly, but the following qualitative description is sufficient:

Proposition. Let $\pi$ be the natural homomorphism of $N$ onto the vector group $N /[N, N]$. If $\gamma \rightarrow U(\gamma)$ is an irreducible unitary representation of $N$, then $U$ is (within equivalence) one of the following two types:

(a) $\chi \circ \pi$, where $\chi$ is a character of $N /[N, N]$;

(b) $(U(\gamma) f)(x)=\alpha(\gamma, x) f(x+\rho(\gamma))$, where the representation space is $L_{C}^{2}\left(R^{p}\right)$ for some $p \geqq 1, \alpha(\gamma, x)$ is a function of absolute value one satisf $y$ ing the usual conditions for a multiplier, and $\rho$ is a homomorphism of $N$ onto $R^{p}$. (Type (a) may be subsumed under type (b) with $p=0$.)

This result is implicit in the work of Dixmier; in particular, it follows easily by induction from his Lemma 12 in [2].

Now let $\Psi$ be the natural projection of $M$ onto the torus $T=N / D[N, N],\left\{\psi_{t}\right\}$ the flow induced on $T$ by $\left\{\gamma_{t}\right\}$.

${ }^{1}$ Research supported by N.S.F. Grant 11287. 
Theorem. $\left\{\phi_{t}\right\}$ is ergodic if and only if the torus flow $\left\{\psi_{t}\right\}$ is ergodic. Every eigenfunction of $V\left(\gamma_{t}\right)$ is of the form $f \cdot \Psi$, where $f$ is an eigenfunction of $\left\{\psi_{t}\right\}$. The remaining spectrum of $V\left(\gamma_{t}\right)$ is Lebesgue.

From this theorem one can describe all ergodic flows of the indicated form. These are necessarily topologically transitive, and, being pointwise almost periodic, minimal. The character of the flow depends only on the rational dependence or independence of some of the Malcev coordinates of $\gamma_{1}$.

These nilflows form an interesting class of minimal dynamical systems midway between the irrational flows on tori, which have pure point spectrum, and the horocycle flows, which are mixing (with Lebesgue spectrum).

Complete proofs of these results will be published elsewhere.

\section{REFERENCES}

1. L. Auslander, F. Hahn, and L. Markus, Topological dynamics on nilmanifolds, Bull. Amer. Math. Soc. vol. 67 (1961) pp. 298-299.

2. J. Dixmier, Sur les réprésentations unitaires des groupes de Lie nilpotents. V, Bull. Soc. Math. France vol. 87 (1959) pp. 65-79.

3. I. M. Gelfand and S. V. Fomin, Geodesic flows on manifolds of constant negative curvature, Uspehi Mat. Nauk (N.S.) vol. 47 (1952) pp. 118-137 (Amer. Math. Soc. Translations Series 2, vol. 1, 1955, pp. 49-66).

4. F. Mautner, Geodesic flows on symmetric Riemann spaces, Ann. of Math. vol. 65 (1957) pp. 416-431.

YALE UNIVERSity AND

UNIVERSITY OF MINNESOTA (IT) 